\title{
Electron Diamagnetic Effect on Axial Force in an Expanding Plasma: Experiments and Theory
}

\author{
Kazunori Takahashi, ${ }^{1,2, *}$ Trevor Lafleur, ${ }^{1}$ Christine Charles, ${ }^{1}$ Peter Alexander, ${ }^{1}$ and Rod W. Boswell ${ }^{1}$ \\ ${ }^{1}$ Space Plasma, Power and Propulsion Laboratory, Research School of Physics and Engineering, \\ The Australian National University, Canberra ACT 0200, Australia \\ ${ }^{2}$ Department of Electrical Engineering and Computer Science, Iwate University, Morioka 020-8551, Japan
}

(Received 16 June 2011; published 28 November 2011)

\begin{abstract}
The axial force imparted from a magnetically expanding current-free plasma is directly measured for three different experimental configurations and compared with a two-dimensional fluid theory. The force component solely resulting from the expanding field is directly measured and identified as an axial force produced by the azimuthal current due to an electron diamagnetic drift and the radial component of the magnetic field. The experimentally measured forces are well described by the theory.
\end{abstract}

DOI: 10.1103/PhysRevLett.107.235001

PACS numbers: 52.30.Ex, 52.72.+v, 52.75.Di

The momentum flux delivered by charged particles in plasmas and its interaction with magnetic fields have been significant subjects associated with astrophysical jets [1], magnetospheric physics [2], thermonuclear fusion devices [3], and electric propulsion systems [4]. It is essential to understand and characterize the gain or loss mechanisms of the momentum flux for clarifying the acceleration or confinement processes of charged particles in nature and in terrestrial plasma devices. For instance, acceleration processes in current-driven plasmas such as applied-field magnetoplasma-dynamic arcjet plasmas have been investigated $[5,6]$. Recent theoretical studies concerning the spontaneous formation of electric double layers (DLs) have shown that no additional net momentum is delivered by the DLs $[7,8]$. Instead, one- and two-dimensional theories have shown enhancements of the net axial force [7,9] from an expanding cross section or an expanding magnetic field (called thrust in the field of electric propulsion), even in current-free plasmas such as radio frequency (rf) inductively coupled or helicon mode discharges. Although direct measurements of the net force from magnetically expanding current-free plasmas are presently under investigation [10-12], the role of the expanding magnetic field on the gain or loss of the axial force in current-free plasmas has not yet been described. In the present Letter, the axial force imparted from the magnetically expanding current-free plasma is directly measured for three different experimental configurations and compared with a two-dimensional fluid theory.

Experiments are performed within the Irukandji vacuum chamber at the Australian National University (ANU), which is $100 \mathrm{~cm}$ in diameter and $140 \mathrm{~cm}$ in length and is pumped down to a base pressure of about $10^{-6}$ Torr. Two rf plasma source systems with an expanding magnetic field are presently used. Figure 1(a) shows a schematic of the first source (custom built at ANU), which has a $25 \mathrm{~cm}$ long, $9 \mathrm{~cm}$ inner diameter Pyrex source tube and an expanding magnetic field applied by using a combination of two axial solenoids located at $z=-5.5 \mathrm{~cm}$ (downstream current,
$I_{B \text { down }}$ ) and $-18.5 \mathrm{~cm}$ (upstream current, $I_{B \text { up }}$ ), where the open exit of the source tube is defined as $z=0$. Axial profiles of the magnetic field $B_{z}$ on axis for solenoidal currents $\left(I_{B}\right.$ up,$\left.I_{B \text { down }}\right)$ of $(6 \mathrm{~A}, 6 \mathrm{~A})$ and $(0 \mathrm{~A}, 6 \mathrm{~A})$ are plotted in Fig. 2(a) and labeled "A mode" and "B mode," respectively. Topologies of the field lines are also drawn in Fig. 2(b). Argon gas is fed into the source tube via a small ceramic tube which is mechanically isolated from the source tube [Fig. 1(a)]. The flow rate is maintained at $25 \mathrm{sccm}$, which gives a pressure within the chamber of about 1 mTorr. A rf loop antenna $(\sim 11 \mathrm{~cm}$ inner diameter and 2 turns) is located at $z=-12 \mathrm{~cm}$ and is mechanically isolated from the source tube. The second source is a permanent magnet (PM) expanding source (custom built at Iwate University) [11], with a similar structure as that shown in Fig. 1(a): briefly, the PM source has a $6.5 \mathrm{~cm}$ inner diameter, $8 \mathrm{~cm}$ long source tube (the cavity length is adjusted using a movable insulating plate located at the closed end of the source) and a combination of two concentric neodymium iron boron PM arrays providing an expanding magnetic field shown in Figs. 2(a) and 2(b) (labeled "C mode"). The rf loop antenna ( $\sim 8 \mathrm{~cm}$ inner diameter and 2 turns) is located at $z=-4.5 \mathrm{~cm}$. For both sources the antennas are powered from a $13.56 \mathrm{MHz}$ rf power generator through an impedance matching circuit.
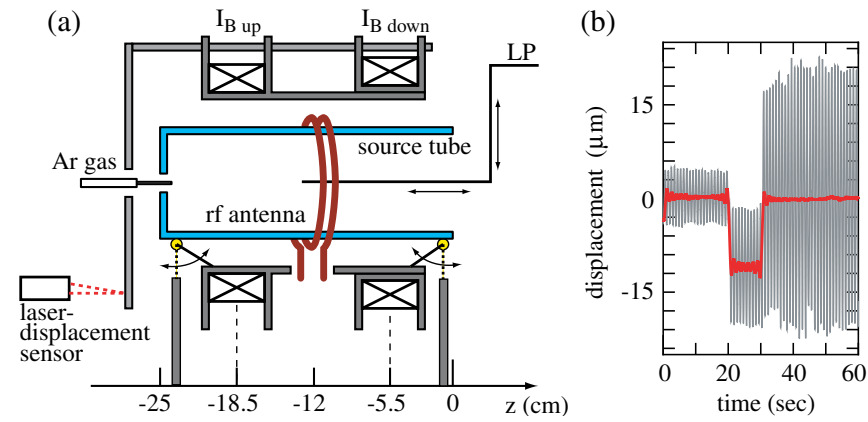

FIG. 1 (color online). (a) Schematic of the experimental setup. (b) Typical displacement signal from the force balance. 
(a)

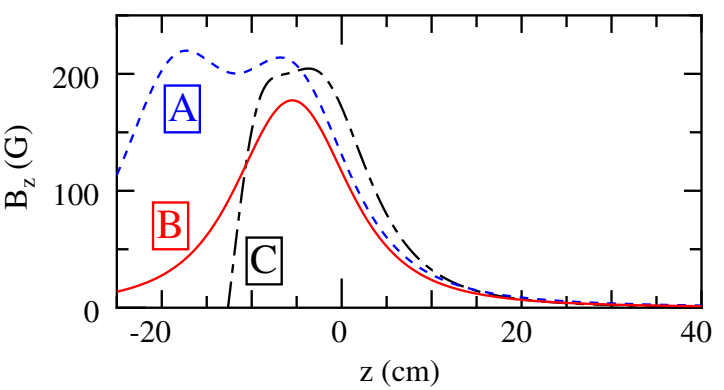

(b)

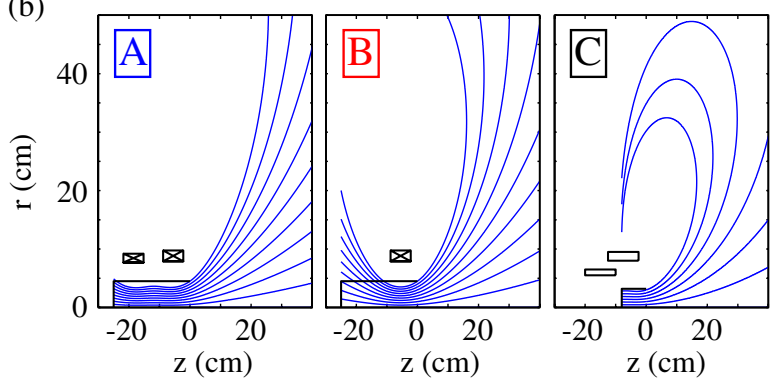

(c)

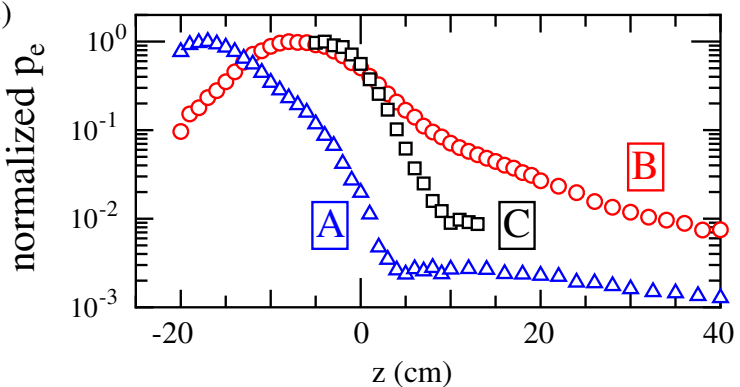

FIG. 2 (color online). (a) Axial profiles of the magnetic field $B_{z}$ on axis for $\left(I_{B \text { up }}, I_{B \text { down }}\right)=(6 \mathrm{~A}, 6 \mathrm{~A})$ (dotted line), (0 A, $6 \mathrm{~A})$ (solid line), and the PMs (dot-dashed line). These are labeled A-C modes, respectively. (b) Topologies of the magnetic field lines. The position of the radial wall of the outer chamber is $r=50 \mathrm{~cm}$. (c) Axial profiles of the normalized electron pressure measured on axis for A mode (open triangles, $470 \mathrm{~W}$ ), B mode (open circles, $540 \mathrm{~W}$ ), and $\mathrm{C}$ mode (open squares, $320 \mathrm{~W})$.

The solenoids or PMs are attached to the force balance immersed within the Irukandji vacuum chamber and their displacement (generated when initiating the plasma) is measured using a high-resolution laser-displacement sensor (resolution, $0.1 \mu \mathrm{m}$; sampling rate, $312.5 \mathrm{~Hz}$ ) [11]. No displacement is detected when the cold gas is introduced; the force by the neutral gas is negligible in the present experiments. The laser system is initially operated with flowing gas and no plasma for a period of $20 \mathrm{~s}$; the rf power and argon plasma are subsequently turned on for about $10 \mathrm{~s}$. After the plasma is turned off, the data are taken for another $30 \mathrm{~s}$. A typical signal of the displacement is plotted in Fig. 1(b) as a thin line. As forces applied to the balance result in an oscillation of the pendulum with a frequency of about $1 \mathrm{~Hz}$ as shown in Fig. 1(b), the data are filtered to remove the pendulum oscillation [bold line in Fig. 1(b)].
The displacement corresponds to the difference between the positions measured during the "plasma off" and "plasma on" periods. The force imparted by the plasma is obtained using the calibration coefficient relating the displacement to the force (typically, $0.5-0.6 \mathrm{mN} / \mu \mathrm{m}$ for the $\mathrm{A}$ and $\mathrm{B}$ modes and $0.35 \mathrm{mN} / \mu \mathrm{m}$ for the $\mathrm{C}$ mode), which can be obtained as described in Ref. [11]. For the A and $\mathrm{B}$ modes of the first source, the measurement of the axial forces are performed when the source tube is attached either to the solenoids or to the Irukandji vacuum chamber. These two distinct configurations, respectively, lead to the direct measurements of the total force $T_{\text {total }}$ resulting from both the source tube and magnetic field, and of the force $T_{B}$ resulting solely from the magnetic field. For the $\mathrm{C}$ mode of the second source, the source tube is attached to the array of PMs and only the measurement of $T_{\text {total }}$ is obtained. The local plasma density $\left(n_{i} \simeq n_{e}=n\right)$ and electron temperature $T_{e}$ are measured with a radially and axially movable Langmuir probe, assuming a Maxwellian electron energy distribution, and using Sheridan's sheath expansion model [13].

Figure 2(c) shows the normalized electron pressure $\left(p_{e}=n k_{B} T_{e}\right)$ derived from the plasma density $n$ and electron temperature $T_{e}$ measured along the $z$ axis, where $k_{B}$ is the Boltzmann constant. The axial positions $z_{0}$ having the maximum electron pressure are at $z_{0}=-17,-7$, and $-4 \mathrm{~cm}$ for the A, B, and $\mathrm{C}$ modes, respectively. The typical plasma density and electron temperature at $(r, z)=\left(0, z_{0}\right)$ for an effective $\mathrm{rf}$ power of about $600 \mathrm{~W}$ are about $4.5 \times 10^{11} \mathrm{~cm}^{-3}$ and $4 \mathrm{eV}, 5 \times 10^{11} \mathrm{~cm}^{-3}$ and $4.5 \mathrm{eV}$, and $1.5 \times 10^{12} \mathrm{~cm}^{-3}$ and $5.5 \mathrm{eV}$ for the $\mathrm{A}, \mathrm{B}$, and $\mathrm{C}$ modes, respectively; the rf antenna current is simultaneously measured with the plasma "on" while performing all experiments to derive the power transfer efficiency [14] and the resultant effective power absorbed into the plasma.

Measurements of $T_{\text {total }}$ and $T_{B}$ are carried out as a function of rf power for cases $\mathrm{A}-\mathrm{C}$ and for cases $\mathrm{A}$ and $\mathrm{B}$, respectively, and the results are shown as open squares and open circles in Fig. 3. The total force $T_{\text {total }}$ increases with the effective $\mathrm{rf}$ power and reaches a maximum of about 2,6 , and $3.5 \mathrm{mN}$ for all the $\mathrm{A}, \mathrm{B}$, and $\mathrm{C}$ modes, respectively. A significant difference in $T_{\text {total }}$ between the A mode and the B mode is observed despite their similar maximum densities, electron temperatures, and source radii. The force $T_{B}$ resulting from the magnetic field increases to about 0.6 and $3 \mathrm{mN}$ for the $\mathrm{A}$ and $\mathrm{B}$ modes, respectively; it is found that the large values for $T_{\text {total }}$ measured for the B mode result from the large values measured for $T_{B}$. The experimental results in Fig. 3 clearly demonstrate the direct measurement of the axial force onto the magnetic field in current-free magnetically expanding plasmas and the subsequent contribution of this force to the total measured force.

The origin of the force onto the magnetic field is now derived from a fluid model. We consider an axisymmetric, 

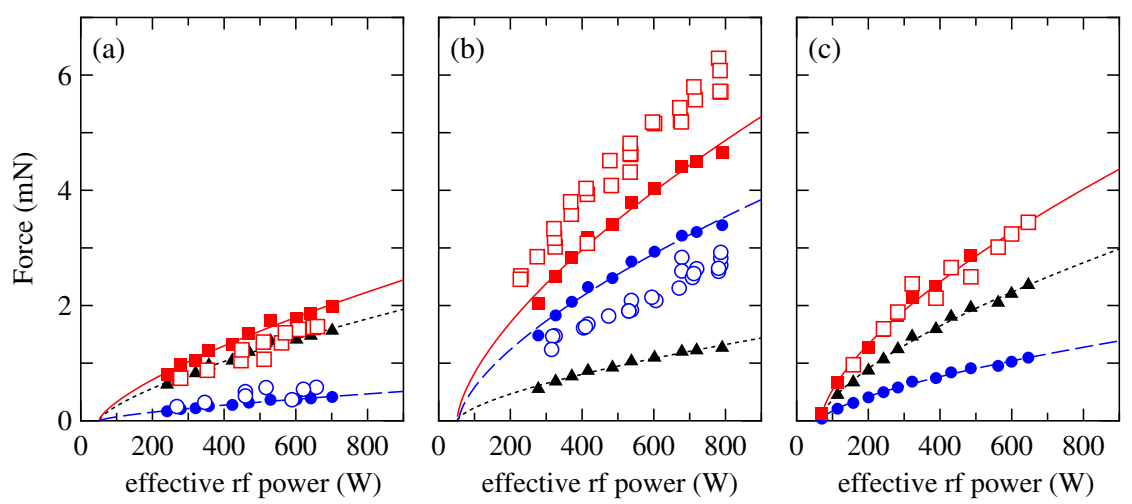

FIG. 3 (color online). Directly measured forces $T_{\text {total }}$ (open squares) and $T_{B}$ (open circles) as a function of the effective rf power for the (a) A mode, (b) B mode, and (c) $\mathrm{C}$ mode, respectively. The forces $T_{s}$ (filled triangles), $T_{B}$ (filled circles), and $T_{\text {total }}$ (filled squares) calculated using Eq. (6) are also plotted together with the fitted curves (dotted, dashed, and solid lines) added as visual guides.

magnetically expanding, current-free plasma system. Assuming quasineutrality, negligible electron inertia, and cold ions, the radial and axial components of momentum equations for electrons and ions in cylindrical coordinates $(r, \theta, z)$ are given by

$$
\begin{gathered}
-e n\left(E_{r}+v_{\theta} B_{z}\right)=\frac{\partial p_{e \perp}}{\partial r}, \\
-e n\left(E_{z}-v_{\theta} B_{r}\right)=\frac{\partial p_{e \|}}{\partial z}, \\
e n\left(E_{r}+u_{\theta} B_{z}\right)=0, \\
e n\left(E_{z}-u_{\theta} B_{r}\right)=\frac{1}{r} \frac{\partial}{\partial r}\left(r m n u_{r} u_{z}\right)+\frac{\partial}{\partial z}\left(m n u_{z}^{2}\right),
\end{gathered}
$$

where $\left(E_{r}, E_{\theta}, E_{z}\right),\left(B_{r}, B_{\theta}, B_{z}\right),\left(v_{r}, v_{\theta}, v_{z}\right),\left(u_{r}, u_{\theta}, u_{z}\right)$, and $n$ are the electric field, magnetic field, electron velocity, ion velocity, and plasma density, which are functions of $(r, z) . e$ is the elementary charge and $m$ is the ion mass. $p_{e \perp}(r, z)=n k_{B} T_{e \perp}$ and $p_{e \|}(r, z)=n k_{B} T_{e \|}$ are the electron pressures perpendicular and parallel to $z$, where $T_{e \perp}$ and $T_{e \|}$ are the electron temperatures perpendicular and parallel to $z$, respectively. The radial component of the ion inertia is also neglected as the ions are mainly accelerated along the $z$ axis in this type of plasma system whether by the DL or by ambipolar axial electric fields $[15,16]$. By eliminating $E_{z}$ from Eqs. (2) and (4), a net axial momentum flux $\tau(r, z) \equiv m n u_{z}^{2}+p_{e \|}$ per unit cross section, which can be regarded as a pressure, can be given as

$$
\frac{\partial \tau}{\partial z}=e n\left(v_{\theta}-u_{\theta}\right) B_{r}-\frac{1}{r} \frac{\partial}{\partial r}\left(r m n u_{r} u_{z}\right),
$$

where the first term on the right-hand side (rhs) is the Lorentz force due to the net azimuthal current and the radial component of the magnetic field. The electron and ion azimuthal currents can be obtained from Eqs. (1) and (3) as $e n v_{\theta}=-e n E_{r} / B_{z}-B_{z}^{-1} \partial\left(p_{e \perp}\right) / \partial r$ and $e n u_{\theta}=$ $-e n E_{r} / B_{z}$, which correspond to the currents from the electron $\mathbf{E} \times \mathbf{B}$ drift, the electron diamagnetic drift, and the ion $\mathbf{E} \times \mathbf{B}$ drift, respectively. Hence, the net current can be expressed by only the electron diamagnetic current as the $\mathbf{E} \times \mathbf{B}$ drifts cannot drive a net current. When the plasma expands with an axially varying plasma radius $r_{p}(z)$, and has zero density at $r \geq r_{p}(z)$, the total axial force $T_{\text {total }}(z)$ is given by the volume integration of $\partial \tau / \partial z$ as

$$
\begin{aligned}
T_{\text {total }}(z)= & T_{s}-2 \pi \int_{z_{0}}^{z} \int_{0}^{r_{p}(z)} r \frac{B_{r}}{B_{z}} \frac{\partial p_{e \perp}}{\partial r} d r d z \\
& -2 \pi \int_{z_{0}}^{z} \int_{0}^{r_{p}(z)} \frac{\partial}{\partial r}\left(r m n u_{r} u_{z}\right) d r d z
\end{aligned}
$$

where $T_{s}=2 \pi \int_{0}^{r_{s}} r p_{e \|}\left(r, z_{0}\right) d r$ originates from the maximum electron pressure [7] and corresponds to the constant of integration along $z$ when integrating from $z_{0}$. As suggested by Fruchtman, this term is conserved along $z$ in the absence of the magnetic field even for collisional plasmas [17]. $r_{s}$ is the source tube radius, and the contributions from the magnetic field and the radial source wall upstream of $z_{0}$ are not included in Eq. (6) for simplicity. The third term on the rhs in Eq. (6) presents an axial force delivered by the ions flowing into the radial source wall; it vanishes as the plasma density is assumed to be zero at the plasma edge. The second term on the rhs in Eq. (6) shows the axial force imparted from the Lorentz force and corresponds to the measured force $T_{B}$ onto the magnetic field (Fig. 3). It results from the azimuthal current $\left[B_{z}^{-1}\left(\partial p_{e \perp} / \partial r\right)\right]$ of the electron diamagnetic drift and the radial component $\left(B_{r}\right)$ of the applied magnetic field. Hence, the present theory clearly identifies the gain (loss) of the net axial force for negative (positive) radial gradient of the electron pressure in an expanding magnetic field in addition to the electron pressure term $T_{s}$ inside the source tube.

To compare the theory with the experiments, $T_{B}, T_{s}$, and $T_{\text {total }}=T_{s}+T_{B}$ are calculated from Eq. (6), together with the following model of the two-dimensional electron pressure $p_{e}(r, z)$ along with the two-dimensional magnetic field $\left(B_{r}, B_{z}\right)$ for the three experimental configurations. To 
model $p_{e}(r, z)$, the plasma radius along $z$ is assumed to be determined by the source tube radius as $r_{p}(z)=r_{s}$ for $z \leq 0$ and by the expanding magnetic field as $r_{p}(z)=$ $r_{s} \sqrt{B_{z}(0,0) / B_{z}(0, z)}$ [18] for $z>0$, and the electron temperature is assumed to be isotropic $\left(T_{e \perp}=T_{e \|}\right)$. The two-dimensional $r-z$ profile of the electron pressure normalized by $p_{e}\left(0, z_{0}\right)$ is modeled as $p_{e}(r, z) / p_{e}\left(0, z_{0}\right)=$ $p_{e n}(0, z) f(r, z)$, where $p_{e n}(0, z)$ and $f(r, z)$ are the normalized electron pressure along $z$ in Fig. 2(c) and a function giving a radial profile of the normalized electron pressure at $z$, respectively. $f(r, z)$ for $r \leq r_{p}$ is given by $f(r, z)=$ $1-\left[r / r_{p}(z)\right]^{a_{1}}$ for the A and C modes, and by $f(r, z)=$ $a_{2}\left\{1-\left[r / r_{p}(z)\right]^{a_{1}}\right\}^{a_{3}}+\left(1-a_{2}\right)\left\{1-\left[r / r_{p}(z)\right]^{a_{4}}\right\}^{a_{5}}$ for the B mode, respectively, and $f(r, z)$ is assumed to be zero for $r>r_{p} . a_{1}-a_{5}$ are fitting parameters of the electron pressure radial profiles measured at $z_{0}$ for $\mathrm{A}-\mathrm{C}$ modes (not shown here), respectively, and remain unchanged along $z$. The values of $a_{1}-a_{5}$ are $a_{1}=5.37$ for A mode, $a_{1}-a_{5}=2.07,3.72,2.16,2.89$, and 4.83 for B mode, and $a_{1}=2.07$ for $\mathrm{C}$ mode, respectively. The maximum electron pressures $p_{e}\left(0, z_{0}\right)$ (plasma density and electron temperature) measured for various if powers are used for the calculation, and the axial integrations in Eq. (6) are performed from $z_{0}$ to the most downstream positions of the electron pressure measurement $(z=40 \mathrm{~cm}$ for A and B modes and $z=13 \mathrm{~cm}$ for $\mathrm{C}$ mode).

The calculated $T_{B}$ (filled circles), $T_{s}$ (filled triangles), and $T_{\text {total }}$ (filled squares) are plotted in Figs. 3(a)-3(c), respectively, where the dashed, dotted, and solid lines are fitted curves added as a visual guide. As is seen, the measured momentum fluxes $T_{B}$ (open circles) and $T_{\text {total }}$ (open squares) are in fairly good agreement with the calculated fluxes (filled circles and squares) for all cases tested. This demonstrates that the axial force in an expanding magnetic field can be enhanced as a result of the momentum gain due to the Lorentz force, a force produced due to an electron diamagnetic drift, and the radial component of the magnetic field. As the downstream electron pressure for B mode is larger than that for the other cases, the contribution from the electron diamagnetic effect is greater and gives the larger $T_{B}$. The larger discrepancy of about 20\% observed between the theoretical and measured $T_{\text {total }}$ and $T_{B}$ for case B could result from the simplifications related to the radial component of the ion inertial term, the axial force delivered onto the radial source wall, and the two-dimensional profile of the electron pressure.

In summary, the direct measurements of the axial force imparted from magnetically expanding current-free plasmas have been performed in three different configurations and compared with a two-dimensional fluid theory. The gain of axial force by the presence of the expanding magnetic field is clearly demonstrated in the experiments. A fluid theory is developed which shows that the axial force is enhanced by the azimuthal current of the electron diamagnetic drift and the radial magnetic field. The directly measured and theoretical forces are in fairly good agreement.

The authors would like to thank Professor A. Fruchtman of the Holon Institute of Technology for many useful discussions.

*kazunori@iwate-u.ac.jp

[1] D. L. Meier, S. Koide, and Y. Uchida, Science 291, 84 (2001).

[2] T. E. Eastman, Geophys. Res. Lett. 3, 685 (1976).

[3] A. G. Peeters, C. Angioni, and D. Strintzi, Phys. Rev. Lett. 98, 265003 (2007).

[4] M. Zuin et al., Phys. Rev. Lett. 92, 225003 (2004).

[5] A. Sasoh, Phys. Plasmas 1, 464 (1994).

[6] H. Tobari, A. Ando, M. Inutake, and K. Hattori, Phys. Plasmas 14, 093507 (2007).

[7] A. Fruchtman, Phys. Rev. Lett. 96, 065002 (2006).

[8] E. Ahedo, Phys. Plasmas 18, 033510 (2011).

[9] E. Ahedo and M. Merino, Phys. Plasmas 17, 073501 (2010).

[10] O. V. Batishchev, IEEE Trans. Plasma Sci. 37, 1563 (2009).

[11] K. Takahashi et al., Appl. Phys. Lett. 98, 141503 (2011).

[12] S. Pottinger, V. Lappas, C. Charles, and R. Boswell, J. Phys. D 44, 235201 (2011).

[13] T. E. Sheridan, Phys. Plasmas 7, 3084 (2000).

[14] T. Lafleur, C. Charles, and R. W. Boswell, J. Phys. D 44, 055202 (2011).

[15] X. Sun et al., Phys. Rev. Lett. 95, 025004 (2005).

[16] B. W. Longmier et al., Plasma Sources Sci. Technol. 20, 015007 (2011).

[17] A. Fruchtman, IEEE Trans. Plasma Sci. 36, 403 (2008).

[18] F. F. Chen, Phys. Plasmas 13, 034502 (2006). 